\section{Assessing effectiveness of regular repositioning in preventing pressure ulcers in children}

\author{
Rifka Putri Andayani,1,2 \\ Nani Nurhaeni, 1 Dessie Wanda1 \\ 1 Faculty of Nursing, Universitas \\ Indonesia; 2Institute of Health Science \\ Mercubaktijaya Padang, Indonesia
}

\begin{abstract}
Pressure ulcers have adverse effects on health. Thus, early detection of damage to skin integrity is important for preventing the occurrence of pressure sores. Meanwhile, two-hourly repositioning is a nursing intervention performed to prevent pressure ulcers. This study aimed to evaluate the implementation of regular repositioning for preventing pressure sores. The Braden Q Scale is an instrument that predicts skin breakdown caused by pressure ulcers. The designs used quasi-experiment pretest and posttest nonequivalent control group; 93 participants were selected through a nonprobability sampling technique by consecutive sampling (43 for the intervention group and 50 for the control group). Respondents in the intervention group were repositioned every 2 hours, and the control group received pressure mattress, which is done for 14 days or until the child can go home. The findings suggest that there is a significant difference in the Braden Q scores from before and after repositioning of the intervention group and the control group using pressure mattress $(\mathrm{P}<0.001)$. Nurses are expected to be able to detect early damage to skin integrity and to implement regular repositioning by using the Braden Q Scale.
\end{abstract}

\section{Introduction}

Pressure ulcers could be affected for health due to their increasing occurrence. Pediatric clients have a risk of pressure ulcers during hospitalization. 1 The prevalence of pressure ulcers in children in the United States (US) was $1.4 \% .{ }^{2}$ In Spain, the prevalence of pressure ulcers in children from 23 hospitals was $3.31 \% ; 1.79 \%$ of these cases happened in pediatric's general wards, and $9.39 \%$ occurred in pediatric intensive care units. ${ }^{3}$ The highest prevalence was in children aged a year $(4.77 \%)$, followed by children $1-3$ years $(2.89 \%){ }^{4}$ From 2013 to 2016 , there were 28 children in Medan who had pressure ulcers; 13 of these children $(46.4 \%)$ were younger than 5 years. In a top referral hospital in Jakarta, the incidence of pressure ulcers in the pediatric units was higher in 2018 (9 children $[0.22 \%])$ than in 2017 (6 children [0.1\%]), and the ulcers mostly appeared on children who had prolonged bed rest.

Pressure ulcers are affected by tissue pressure and tolerance. Pressure intensity and the duration of being in a certain position could increase the risk of developing pressure ulcers. ${ }^{5}$ Meanwhile, tissue tolerance is affected by age, tissue, medicines, nutritional status, medical diagnosis, body temperature, and physical condition. ${ }^{1}$ Some intrinsic and extrinsic factors need to be taken into account in assessing tissue tolerance. ${ }^{1}$ The intrinsic factors include age, nutrition, oxygenation, and tissue perfusion, and the extrinsic factors include friction, skin moisture, and the equipment used. ${ }^{1}$

Pressure ulcers require a long healing process and affect children's emotions. Pediatric clients might have the risk of infection, prolonged hospital stay, decreased rest time, and skin breakdown due to topical medication, which negatively impact children's mental development. ${ }^{6}$ and increase the cost of hospitalization. ${ }^{7}$ The increased incidence of pressure ulcers affects the medical expenses of clients and health care systems. ${ }^{8-11}$

Preventing pressure ulcers and repairing damaged tissue integrity are the main focus with regard to health services. Initial assessments are expected to prevent pressure ulcers. Early detection could be conducted using the Braden Q Scale, which is an instrument that assesses the risk of pressure ulcers and predicts risky and risk-free clients. ${ }^{1,9}$ Common preventive interventions for children's treatment include the use of gel/water pillows and decubitus beds and repositioning. ${ }^{12}$

Regular repositioning could reduce pressure and prevent prolonged ischemia. ${ }^{13}$ In addition, it could be the most effective way to prevent pressure ulcers because it could modify pressure sores. ${ }^{5}$ Repositioning every two hours effectively reduced pressure ulcer incidences by $14 \% .{ }^{14}$ Two-hourly repositioning should be performed regularly within 24 hours, and it requires cooperation from patients' parents. ${ }^{15}$ Children highly depend on their parents psychologically; therefore, parents' help is needed in order to provide a convenient ambiance for the children. Such prevention could be performed by reducing the pressure around bony prominences and paying attention to the children's comfort, such as by using pillows and cotton and by hand folding, given that repositioning can be
Correspondence: Nani Nurhaeni, Pediatric Nursing Department, Faculty of Nursing, Universitas Indonesia, Jalan Prof. Dr. Bahder Djohan, Kampus UI Depok, West Java, 16424 Indonesia.

Tel.: +6221.78849120 - Fax: +6221.7864124.

E-mail: nani-n@ui.ac.id

Key words: Braden Q; pressure ulcer; repositioning.

Acknowledgments: The writer expresses gratitude to all respondents and their families, the building A nurses at Dr. Cipto Mangunkusumo Hospital Jakarta.

Funding: This study was supported by Hibah PITTA 2019, funded by DRPM Universitas Indonesia (No. NKB-0496/UN2.R3.1/HKP.05. 00/2019)

Contributions: RPA, NN and DW conceived the idea and plan of research. RPA, NN and DW contributed to the design and implementation of the research also verified the analytical method. RPA wrote the manuscript in consultation with $\mathrm{NN}$ and DW. All authors discussed the results and reviewed the final manuscript.

Ethical approval: Ethical clearance was issued by the Research Ethics Committee of the Faculty of Nursing Universitas Indonesia (No. 38/UN2.F12.D/HKP.02.04/2019).

Conflict of interest: The authors have no conflict of interest.

This work is licensed under a Creative Commons Attribution NonCommercial 4.0 License (CC BY-NC 4.0)

(C) Copyright: the Author(s), 2020

Licensee PAGEPress, Italy

Pediatric Reports 2020; 12(s1):8696

doi:10.4081/pr.2020.8696

uncomfortable. ${ }^{16}$ Children would experience friction if repositioning is not performed correctly. Considering this background, this work used the Braden Q Scale to study the implementation of repositioning to prevent pressure ulcers in children.

\section{Materials and Methods}

The study designs were quasi-experiment pre-test and post-test nonequivalent control group. The researcher assigned respondents into intervention and control groups. The intervention group was children who were treated in an acute room who underwent regular repositioning with 2hourly repositioning should be given regularly within 24 hours, while the control 
group was children who were treated in a non-infectious room received standard intervention in pediatric units using pressure mattress. The study population comprised pediatric patients on the pediatric room and the respondents were selected through a nonprobability sampling technique by consecutive sampling. The inclusion criteria were being aged between 1 month and 18 years and having a treatment length of 24 hours. Children who were anxious, lacked cooperation, edema, and had previous pressure ulcers were excluded. The total sample of this innovation project involved 93 children. This research was conducted in the pediatric area within 10 weeks (January 28, 2019, to April 5, 2019).

The instrument used in this study to collect data on the respondents' characteristics was an observation checklist that covered age, sex, parents' socio-economy and education, two-hourly repositioning records, Braden Q scores, and nutritional status. The data analysis comprised univariate and bivariate analyses. The bivariate analysis involved an independent $t$ test and a paired t test. Ethical clearance was issued by the Ethical Committee of the Faculty of Nursing of Universitas Indonesia (No. 38/UN2.F12.D/HKP.02.04/2019).

\section{Results}

Table 1 shows that the respondents in the intervention group were younger by 47.42 months than those in the control group.

Table 2 shows that the respondents in the intervention group were mostly females (23 respondents [53.5\%]), and the most common medical diagnosis was on the respiratory system (12 respondents [27.9\%]). Meanwhile, the control group was dominated by male respondents (31 respondents $[62 \%])$, and the most common medical diagnosis was on the musculoskeletal system (21 respondents [42\%]). That most of the parents earned $\geq$ IDR 2,000,000 (34 respondents in the intervention group [79.1\%] and 49 respondents in the control group [98\%]). The respondent distribution based on education shows that most of the mothers were senior high school (SMA) graduates (26 respondents in the intervention group [60.5\%] and 38 respondents in the control group [76\%]). In addition, most of the parents were private/BUMN employees $(20$ respondents in the intervention group [46.5\%] and 31 respondents in the control group [62\%]).

Table 3 shows the mean scores of the respondents' characteristics based on their Braden Q scores before and after the interven- tion. The Braden Q scores for the intervention group before and after intervention were 16.77 and 18.77 , respectively, and the corresponding Braden Q scores for the control group were 20.02 and 20.64, respectively.

Table 4 shows a significant difference in the Braden Q scores obtained before and after repositioning in the intervention group and before and after the standard intervention in the control group $(\mathrm{P}<0.05)$.

Table 5 shows a significant difference in the Braden Q scores obtained after repositioning in the intervention group and after standard intervention in the control group $(\mathrm{P}=0.002 ; \alpha=0.05)$.

\section{Discussion}

Age can determine one's health condition. In this innovation project, the average ages of the intervention and control groups were 47.42 and 75.48 months, respectively. The average age applied in this study was similar to that in a previous study that stated that pressure ulcers frequently happened to children aged 5.9 years and prevalent in children older than 3 years. 4,17 The intervention group of this innovation project was dominated by females (23 respondents [53.5\%]), and the most common medical diagnosis was pneumonia (12 respondents $[27.9 \%])$. Meanwhile, the control group was dominated by male respondents ( 31 respondents $[62 \%]$ ), and the most frequent medical diagnosis was osteosarcoma (14 respondents [28\%]). In previous research, from 65,359 children aged $0-18$ years who experienced pressure ulcers, $56.7 \%$ were males, and $43.3 \%$ were females. ${ }^{4}$ Children aged $0-2$ years, who have limited communication skills, had more cases of pressure ulcers than did other age groups. Such increased risk of pressure ulcers is often

Table 1. Respodents' age.

\begin{tabular}{lccccc} 
Respondents' characteristics & Mean & SD & Min & Max & $95 \%$ CI \\
Age & & & & & \\
Intervention & 47.42 & 60.297 & 2 & 208 & $28.86-65.98$ \\
Control & 75.48 & 60.171 & 2 & 215 & $58.38-92.58$ \\
\hline
\end{tabular}

Table 2. Characteristics based on sex, medical diagnosis, parents' socio-economy, education, and occupation.

\begin{tabular}{lcccc} 
Respondents' characteristics & \multicolumn{3}{c}{ Frequency } \\
Sex & Intervention & $\%$ & Control & $\%$ \\
Female & 23 & 53.5 & 19 & 38 \\
Male & 20 & 46.5 & 31 & 62 \\
Medical diagnosis & & & & \\
Respiratory system & 12 & 27.9 & 3 & 6 \\
Digestive system & 9 & 20.93 & 1 & 2 \\
Urinary system & 4 & 9.3 & 21 & 42 \\
Musculoskeletal system & 1 & 2.3 & 7 & 14 \\
Nervous system & 7 & 16.3 & 14 & 28 \\
Immune system & 6 & 14 & 4 & 8 \\
Cardiovascular system & 3 & 6.97 & & \\
Sensing system & 1 & 2.3 & & \\
\hline Socio-economy & & & & \\
< Rp 2,000,000 & 9 & 20.9 & 1 & 2 \\
$\geq$ Rp 2,000,000 & 34 & 79.1 & 49 & 98 \\
Mother's education & & & & \\
Elementary and junior high school & 8 & 18.6 & 6 & 12 \\
Senior high school & 26 & 60.5 & 38 & 76 \\
Higher education & 9 & 20.9 & 6 & 12 \\
\hline Occupation & & & & \\
Unemployed & 3 & 7 & 1 & 2 \\
Civil servant & 6 & 14 & 4 & 8 \\
Private/BuMN employee & 20 & 46.5 & 31 & 62 \\
Laborer & 5 & 11.6 & 1 & 2 \\
Entrepreneur & 8 & 18.6 & 12 & 24 \\
Fisherman & 1 & 2.3 & 1 & 2 \\
\hline
\end{tabular}


observed in children with limited communication capabilities, such as neonates, infants, toddlers, and children with neurological problems. 7,18

Pressure ulcers appear on the outermost layer of the skin due to external pressure and can expand into the deeper layers.7,19 The Braden Q Scale is an instrument that assesses the risk of pressure ulcers. It can predict the possibility of pressure ulcer occurrences based on the recommendation from the National Pressure Ulcer Advisory Panel (NPUAP), which covers mobility, activity, moisture, friction, nutrition, oxygenation, tissue perfusion, and sensory perception. According to the distribution table of the intervention group's results, the average Braden Q scores were 16.77 and 18.47 before and after repositioning, respectively. This condition was similar to that of the control group, wherein the Braden Q scores before and after treatment were 20.02 and 20.64 , respectively. These findings show an increase in the Braden Q scores, which meant decreased risk of pressure ulcers.

The medical condition also affects the Braden Q score. In this evidence-based practice, children who suffered from neurological problems, such as encephalitis and hydrocephalus, had lower Braden Q scores compared with children who suffered from diarrhea, pneumonia, febrile, pericardial effusion, urinary tract infection, and other infectious diseases. The occurrences of pressure ulcers were caused by a lack of mobility, sensory perception, and mother's or caregiver's knowledge about neurological problems. Repositioning is a common nursing intervention that can be performed by nurses independently to prevent the risk of skin breakdown, especially in children who are immobile due to neurological problems, such as encephalitis and hydrocephalus. These children suffer from motor skills disorders and need their nurses and parents to perform repositioning every two hours to prevent pressure ulcers. 16

The risk factors that could cause pressure ulcers were divided into two types, namely, intrinsic and extrinsic factors. Several steps need to be conducted to reduce the impacts of risk factors, such as assessing the risk of pressure ulcers and implementing preventive actions (skin treatment and pressure management). The Braden Q Scale is recommended for assessing the risk of pressure ulcers. ${ }^{20}$ This instrument can predict the occurrence of pressure ulcers. ${ }^{1}$

There are many interventions designed to prevent pressure ulcers in children; such measures include using supporting surfaces (like bed, integrated bed system, sheet, and pillow), repositioning, improving nutrition, applying skin treatment, and using topical creams. ${ }^{21}$ One of the nursing interventions implemented in this evidence-based practice was repositioning. Regular repositioning can reduce pressure and the likelihood of prolonged ischemia. ${ }^{13}$ Pressure ulcers can be prevented by reducing the pressure on bony prominences while paying attention to the children's comfort, such as by using pillows, cotton, and hand folding. ${ }^{16}$ Psychologically, children highly depend on their parents; therefore, good cooperation from parents is required to provide a safe and comfortable situation for child patients. 16

In this evidence-based practice, twohourly repositioning effectively improved the Braden Q scores. There was a significant difference in the Braden Q scores before and after treatment (16.77 and 18.47, respectively, in the intervention group $[\mathrm{P}<0.05]$ and 20.02 and 20.64, respectively, in the control group $[\mathrm{P}<0.05])$. Although both groups showed significant differences, the control group showed a larger gap (1.7) than did the intervention group (0.62). Such conditions happened because the lengths of stay in the control group were shorter than those in the intervention group. Some respondents in the control group were hospitalized for chemotherapy and allowed to return home unless the chemotherapy had any adverse effect, thereby reducing the risk of developing pressure ulcers. There were also clients in the control group who had decreased Braden $\mathrm{Q}$ scores due to deteriorated physical condition, i.e., many bony prominences and resulting high risk of pressure ulcers. Pressure ulcers commonly appeared on the sacrum area after hours of sitting on chairs, wheelchairs, and beds. ${ }^{22}$ Gel or foam pillows cannot redistribute pressure until a certain time, thereby failing to properly prevent the risk of pressure ulcers. ${ }^{22}$

Table 3. Respondents' Braden Q Score.

\begin{tabular}{lcc} 
Group & Mean \pm SD & $95 \% \mathrm{CI}$ \\
Intervention & & \\
Before & $16.77 \pm 2.707$ & $15.93-17.60$ \\
After & $18.47 \pm 3.034$ & $17.53-19.40$ \\
\hline Control & & \\
Before & $20.02 \pm 3.365$ & $19.06-20.98$ \\
After & $20.64 \pm 3.457$ & $19.66-21.62$ \\
\hline
\end{tabular}

Table 4. Differences in Braden Q Scores before and after repositioning.

\begin{tabular}{|c|c|c|c|c|c|c|c|c|}
\hline Variable & & tervent & & $\mathbf{P}$ & & Contro & & $\mathbf{P}$ \\
\hline & Mean & SD & $95 \%$ CI & & Mean & SD & $95 \% \mathrm{CI}$ & \\
\hline Braden C & & & & & & & & \\
\hline Before & 16.77 & 2.707 & $-2.289 ;-1.106$ & $<0.001^{*}$ & 20.02 & 3.365 & $-0.954 ;-0.286$ & $<0.001^{*}$ \\
\hline After & 18.47 & 3.034 & & & 20.64 & 3.457 & & \\
\hline
\end{tabular}

*Significant at $\mathrm{P}<0.05$.

Table 5. Braden Q Scores after repositioning.

\begin{tabular}{lccccc} 
Variable & \multicolumn{2}{c}{ Intervention } & \multicolumn{2}{c}{ Control } & 95\% CI \\
& Mean & SD & Mean & SD \\
Braden Q Score & 18.47 & 3.034 & 20.64 & 3.457 & $-3.525 ;-0.825$ \\
\hline * & & & $0.002 *$ \\
\hline
\end{tabular}

* Significant at $\mathrm{P}<0.05$. 
2018;19:29-37.

Children who are required to be on bed rest are made to lie on decubitus beds. Administering decubitus bed treatment does not mean that the nurses, in giving of comprehensive care, should ignore the risk of pressure ulcers. The control group yielded low Braden Q scores, which meant increased risk of pressure ulcers. Therefore, providing a decubitus bed is insufficient; two-hourly repositioning should be performed while still paying attention to patient comfort in order to prevent the risk of pressure ulcers in children.

\section{Conclusions}

The implementation of two-hourly repositioning effectively increases Braden Q scores and can thus be conducted to prevent hospitalized children from developing pressure ulcers. The results of this evidence-based practice can be implemented as a nursing intervention for preventing pressure ulcers in children. Limitations of the study require cooperation from parents to 2-hourly repositioning should be given regularly within 24 hours.

\section{References}

1. Noonan C, Quigley S, Curley MAQ. Using the Braden Q scale to predict pressure ulcer risk in pediatric patients. J Pediatr Nurs 2011;26:566-75.

2. Razmus I, Bergquist-Beringer $\mathrm{S}$. Pressure injury prevalence and the rate of hospital-acquired pressure injury among pediatric patients in acute care. $\mathrm{J}$ Wound Ostomy Continence Nurs 2017; 44:110-7.

3. Pancorbo-Hidalgo PL, Torra-Bou JE, Garcia-Fernandez FP, SoldevillaAgreda JJ. Prevalence of pressure injuries and other dependence-related skin lesions among paediatric patients in hospitals in Spain. EWMA Journal
4. Sánchez-Lorente MM, SanchisSánchez E, García-Molina P, et al. Prevalence of pressure ulcers in the paediatric population and in primary health care: An epidemiological study conducted in Spain. J Tissue Viability 2018; 27:221-5.

5. Romanelli M, Clark M, Gafen A, Ciprandi G. Science and practice of pressure ulcer management. London, UK: Springer; 2018.

6. García-Molina P, Balaguer-López E, García-Fernández FP, et al. Pressure ulcers' incidence, preventive measures, and risk factors in neonatal intensive care and intermediate care units. Int Wound J 2018;15:571-9.

7. Schluer AB. Pressure ulcers in maturing skin - a clinical perspective. J Tissue Viability 2017;26:2-5.

8. Demarré L, Van Lancker A, Van Hecke A, et al. The cost of prevention and treatment of pressure ulcers: A systematic review. Int J Nurs Stud 2015;52: 1754-74.

9. Liao Y, Gao G, Mo L. Predictive accuracy of the Braden Q Scale in risk assessment for paediatric pressure ulcer: A meta-analysis. Int J Nurs Sci 2018;5:419-26.

10. Powers, J, Ames, C. Take action to solve causes of pressure injuries. In pressure injuries: Prevention across the acute care continuum. American Nurse Today [Internet]; 2018:4-6. Available from: https://www.myamericannurse. com/wp-content/uploads/2018/05/ DabirSupplement_May2018.pdf

11. Whitty JA, McInnes E, Bucknall T, et al. The cost effectiveness of a patient centred pressure ulcer prevention care bundle: Findings from the INTACT cluster randomised trial. Int J Nurs Stud 2017;75:35-42.

12. Riccioni N, Berlanga R, Hagan J, et al. Interrater reliability of the Braden and
Braden Q by Skin Champion nurses. J Pediatr Nurs 2019;44:9-15.

13. Sarsak HI. Review of pressure ulcers management in pediatrics: assessment, prevention, and intervention. J Pediatr Neonatal Care 2018;8:245-8.

14. Bergquist-Beringer S, Dong L, He J, Dunton N. Pressure ulcers and prevention among acute care hospitals in the United States. Jt Comm J Qual Patient Saf 2013;39:404-14.

15. Fletcher J. Reposition patients effectively to prevent pressure ulcers. Wounds International 2017;8:7-10.

16. Devi SJ, Kaur S, Kaur S. Effect of comfort measures and repositioning on prevention of pressure ulcers in bedridden children. Nursing and Midwifery Research Journal 2013;9:99-107.

17. Jackson D, Sarki AM, Betteridge R, Brooke J. Medical device-related pressure ulcers: A systematic review and meta-analysis. Int J Nurs Stud 2019; 92:109-20.

18. Haimy A, Kopplin K, Gefen, A. Device related pressure ulcers from a biomechanical persperctive. Comput Method Biomec 2018;37-41.

19. Lam C, Elkbuli A, Benson B, et al. Implementing a novel guideline to prevent hospital-acquired pressure ulcers in a trauma population: A patient-safety approach. J Am Coll Surgeons 2018; 226:1122-7.

20. Curley MA, Quigley SM, Lin M. Pressure ulcers in pediatric intensive care: Incidence and associated factors. Pediatr Crit Care Me 2003;4:284-90.

21. Qaseem A, Mir TP, Starkey M, Denberg TD. Risk assessment and prevention of pressure ulcers: A clinical practice guideline from the American College of Physicians. Ann Intern Med 2015;162: 359-69.

22. Anders J, Heinemann A, Leffmann C, et al. Decubitus ulcers: Pathophysiology and primary prevention. Dtsch Arztebl Int 2010;107:371-82. 